Submitted on June 6, 2020.

\title{
Metabolism of phosphorus compoundsand taxonomic position of the Aspergillus niger AM1 mold
}

\author{
(C) Anton Z. Mindubaev, ${ }^{1{ }^{*+}}$ Edward V. Babynin, ${ }^{3}$ Elena K. Badeeva,${ }^{2}$ SalimaT. Minzanova,${ }^{2}$ \\ Lubov' G. Mironova, ${ }^{2}$ Ilias S. Nizamov, ${ }^{3}$ Khasan R. Khayarov, ${ }^{3}$ and Akosah Yaw Abayie ${ }^{3}$ \\ ${ }^{1}$ Institute of Power Engineering and Advanced Technologies.FRC Kazan Scientific Center, Russian Academy \\ of Sciences.E-mail:mindubaev@iopc.ru; mindubaev-az@yandex.ru \\ ${ }^{2}$ Institute of Organic and Physical Chemistry named after A.E. Arbuzov. Kazan Scientific Center \\ of the Russian Academy of Sciences. Arbuzov St., 8. Kazan, 420088. Republic of Tatarstan. Russia. \\ ${ }^{3}$ Kazan (Volga Region) Federal University. University St., 18.Kazan, 420008.Republic of Tatarstan. Russia.
}

*Supervising author; ${ }^{+}$Corresponding author

Keywords: detoxication, phosphorus compounds, Aspergillus niger, phylogenetic tree.

\section{Abstract}

White phosphorus is one of the most dangerous environmental pollutants. However, it is used in industry and for military purposes; therefore, it is impossible to overlook the fact that this substance is constantly released into the environment. In our works, cultures of microorganisms growing in media with a content of white phosphorus up to $1 \%$ were obtained for the first time. This exceeds the TLV in wastewater by 5000 times! These cultures are unique, and they are only in our possession. For the first time, cultures were grown in media containing white phosphorus as the sole source of phosphorus. In these environments, microorganisms grew without experiencing phosphorus starvation. That is, they oxidized white phosphorus to phosphate, which is necessary for vital activity! This is first ever example of the inclusion of white phosphorus in the biospheric circulation of the phosphorus element.

It turned out that microorganisms that neutralize elemental phosphorus are able to biodegrade most of the spectrum of phosphorus compounds. Our studies of the metabolism of phosphorus-containing compounds of various classes confirm this. Since the chemistry of phosphorus is diverse, it is necessary to collect significant material on the metabolism of many classes of compounds. In this article, we describe the continuation of this work. It turned out that Aspergillus niger AM1 is able to utilize dithiophosphate of the simplest structure as sources of phosphorus, but is not able to utilize substituted dithiophosphonate. In addition, in the present work, we clarified the previously obtained results on the metabolism of phosphoric acid ester and phosphoramide.The NMR method demonstrated that $A$. niger AM1 slowly metabolizes hypophosphite resulting from the biodegradation of white phosphorus, but does not metabolize phosphite. The NMR data conforms to fungal growth dynamics with these substances in media. Also, was first studied phylogenetic relationship of $A$. niger AM1 with biodegradable A. niger and A. bombycis strains from the NCBI database.

\section{References}

[1] L. Pauling. The carbon-carbon triple bond and the nitrogen-nitrogen triple bond. Tetrahedron. 1962. Vol.17. No.3-4. P.229-233.

[2] M.I. Eremets, A.G. Gavriliuk, I.A. Trojan, D.A. Dzivenko, R. Boehler. Single-bonded cubic form of nitrogen. Nature Materials. 2004.Vol.3. P.558-563.

[3] M. Ruck, D. Hoppe, B. Wahl, P. Simon, Y. Wang, G.Seifert. Fibrous red phosphorus. Angew Chem Int Ed Engl. 2005. Vol.44. No.46. P.7616-7619.

[4] N.A. Piro, J.S. Figueroa, J.T. McKellar, C.C. Cummins. Triple-Bond Reactivity of Diphosphorus Molecules. Sciences. 2006. Vol.313. No.5791. P.1276-1279.

[5] A.E. Seitz, F. Hippauf, W. Kremer, S. Kaskel, M. Scheer. Facile storage and release of white phosphorus and yellow arsenic. Nature communications. 2018. Vol.9. No.361. P.1-6.

[6] D. Tofan, C.C. Cummins. Photochemical Incorporation of Diphosphorus Units into Organic Molecules. Angew Chem Int Ed. 2010. Vol.49. No.41. P.7516-7518.

[7] C. Mealli, A. Ienco, M. Peruzzini, G. Manca. The atomic level mechanism of white phosphorous demolition by di-iodine. Dalton Trans. 2018. Vol.47. No.2. P.394-408. 
[8] W.W. Schoeller, G.D. Frey. White Phosphorus Degradation with a NacNac Aluminum Carbene Analogue: The Biradical Reaction Mechanism. Inorg. Chem. 2014. Vol.53. No.10. P.4840-4846.

[9] J. Li, Y.Jin, M. Chen, J. Li. A novel green and safe dearsenization of white phosphorus by selective electrocatalytic oxidation. Separation and Purification Technology. 2019. Vol.213. P.314-321.

[10] D. Kaposi. On the possibility of critiquing Israel: The Times' engagement with Israel's deployment of white phosphorous during the first Gaza war. Media, War \& Conflict. 2016. Vol.9. No.3. P.272-289.

[11] J.R. Choi, K.W. Yong, J.Y. Choi, A. Nilghaz, Y. Lin, J. Xu, X. Lu. Black Phosphorus and its Biomedical Applications. Theranostics. 2018. Vol.8. No.4. P.1005-1026.

[12] W.A. Spitzig. The Effects of Phosphorus on the Mechanical Properties of Low-Carbon Iron. Metallurgical Transactions. 1972. Vol.3. No.5. P.1183-1188.

[13] V.N. Antsiferov, A.A. Shatsov, S.A. Oglezneva. Sintered metals and alloys. Structure and properties of powder metallurgy phosphorous steels. Powder Metallurgy and Metal Ceramics. 1999. Vol.38. No.3-4. P.162-165.

[14] M.O. Durowoju, I.A. Babatunde. The Effect of Varying the Composition of Phosphorus on the Microstructure and Mechanical Properties of Tin-Bronze Alloys. International Journal of Modern Engineering Research (IJMER). 2013. Vol.3. No.3. P.1801-1804.

[15] B.A. MacKay, M.D. Fryzuk. Dinitrogen Coordination Chemistry: On the Biomimetic Borderlands. Chem. Rev. 2004.Vol.104. No.2.P.385-401.

[16] I. Franzoni, L. Guénée, C. Mazet. Chiral monodentate phosphine ligands for the enantioselective $\alpha$-and $\gamma$-arylation of aldehydes. Tetrahedron. 2014. Vol.70. No.27-28. P.4181-4190.

[17] J.V. Carey. Synthesis of homochiral mono- and bis-phosphine ligands for homogeneous catalysis. Chimica Oggi - Chemistry Today. 2014. Vol.32. No.2. P.45-50.

[18] L.M. Groves, B.D. Ward, P.D. Newman, P.N. Horton, S.J. Coles, S.J.A. Pope. Synthesis and characterisation of fluorescent aminophosphines and their coordination to gold(I). Dalton Trans. 2018. Vol.47. No.28. P.9324-9333.

[19] M. Echeverri, A. Alvarez-Valdés, F. Navas, J. Perles, I. Sánchez-Pérez, A.G. Quiroga. Using phosphine ligands with a biological role to modulate reactivity in novel platinum complexes. $R$. Soc. opensci. 2018. Vol.5. No.171340. P.1-12.

[20] H.N. Schulz, H.D. Schulz. Large Sulfur Bacteria and the Formation of Phosphorite. Science. 2005. Vol.307. No.5708. P.416-418.

[21] J.S. Compton, E.W. Bergh. Phosphorite deposits on the Namibian shelf. Marine Geology. 2016. Vol.380.No.1. P.290-314.

[22] M.P. Robertson, G.F. Joyce. The Origins of the RNA World. Cold Spring Harb Perspect Biol. 2012. Vol.4. No.5. a003608. P.1-22.

[23] A. Vázquez-Salazar, A. Lazcano. Early Life: Embracing the RNA World. Current Biology. 2018. Vol.28. No.5. P.R208-R231.

[24] J.D. Tonera, D.C. Catling. A carbonate-rich lake solution to the phosphate problem of the origin of life. PNAS. 2019. P.1-6.

[25] M. Arribat, F. Cavelier, E. Rémond. Phosphorus-containing amino acids with a $\mathrm{P}-\mathrm{C}$ bond in the side chain or a $\mathrm{P}-\mathrm{O}, \mathrm{P}-\mathrm{S}$ or $\mathrm{P}-\mathrm{N}$ bond: from synthesis to applications. $R S C A d v \mathbf{2 0 2 0}$. Vol.10. No.6678. P.6678-6724.

[26] V.E. Viola. Nucleosynthesis of the chemical elements. Treb. Soc. Cat. Biol. 1986. Vol.39. P.49-71.

[27] G. Cescutti, F. Matteucci, E.Caffau, P. François. Chemical evolution of the Milky Way: the origin of phosphorus. $A \& A$. 2012.Vol.540. No.A33. P.1-4.

[28] J.W. Swensson. Phosphorus Lines in the Solar Spectrum. Zeitschrift für Astrophysik. 1966. Vol.64. P.11-15.

[29] A.Z. Mindubaev, D.G. Yakhvarov. Biodegradation as a method for waste processing: view on the problem. Part 1. The essence of the method. Butlerov Communications. 2013. Vol.33. No.3. P.1-37. ROI: jbc-02/13-33-3-1

[30] A.Z. Mindubaev, D.G. Yakhvarov. Biodegradation as a method for waste processing: view on the problem. Part 2. Are xenobiotics really xenobiotics? Butlerov Communications. 2013. Vol.34. No.4. P.1-20. ROI: jbc-02/13-34-4-1

[31] A.Z. Mindubaev. Biodegradation of xenobiotics as self-preservation of nature. Biomolekula. 2017.https://biomolecula.ru/articles/biodegradatsiia-ksenobiotikov-kak-samozashchita-prirody

[32] A.Z. Mindubaev. Biodegradation of toxic substances as self-protection of nature. The North Caucasus Ecological Herald. 2018. Vol.14. No.1. P.11-23. (russian)

[33] A.Z. Mindubaev. Who ate the polyethylene? Science and Life. 2018. Vol.4. P.32-38. (russian) 
[34] A.Z. Mindubaev. Are xenobiotics really xenobiotics? One aspect of the diversity of natural compounds. Biomolekula. 2018. https://biomolecula.ru/articles/iavliaiutsia-li-ksenobiotiki-ksenobiotikami-odna-izstoron-raznoobraziia-prirodnykh-soedinenii

[35] A.Z. Mindubaev. Reflections on natural and unnatural chemistry. Biomolekula. Раздумья о природной и неприродной химии. Биомолекула. 2019. https://biomolecula.ru/articles/razdumia-o-prirodnoi-ineprirodnoi-khimii

[36] A.Z. Mindubaev. Microorganisms destructors and their role in the purification of natural environments (review). Living and bio-inert systems. 2020. No.31. P.1-20. (russian)

[37] A.Z. Mindubaev. Microbes as orderlies. Nauka I zhizn. 2020. No.4. P.28-32. (russian)

[38] A.S. Sirotkin, G.I. Shaginurova, K.G. Ippolitov. Aggregation of microorganisms: floccules, biofilms, microbial granules. Kazan: Phen. 2007. P. 159.(russian)

[39] R.L. Singh. Principles and Applications of Environmental Biotechnology for a Sustainable Future. Applied Environmental Science and Engineering for a Sustainable Future. Springer Singapore. 2017. $502 p$.

[40] S.N. Singh. Microbial Degradation of Xenobiotics. Springer-Verlag Berlin Heidelberg. 2012. 486p.

[41] L.B.M. Ellis, L.P. Wackett. Use of the University of Minnesota Biocatalysis/Biodegradation Database for study of microbial degradation. Microbial Informatics and Experimentation. 2012. Vol.2. No.1. P.1-10.

[42] J. Gao, L.B.M. Ellis, L.P. Wackett. The University of Minnesota Biocatalysis/Biodegradation Database: improving public access. Nucleic Acids Research.2010. Vol.38. P.D488-D491.

[43] J. Bordoloi, H.P.D. Boruah. Analysis of Recent Patenting Activities in the Field of Bioremediation of Petroleum Hydrocarbon Pollutants Present in the Environment. Recent Pat Biotechnol. 2018. Vol.12. No.1. P.3-20.

[44] H.D.M. Villela, R.S. Peixoto, A.U. Soriano, F.L. Carmo. Microbial bioremediation of oil contaminated seawater: A survey of patent deposits and the characterization of the top genera applied. Sci Total Environ. 2019. Vol.666. P.743-758.

[45] X.F. Sheng, F. Zhao, L.Y. He, G. Qiu, L. Chen. Isolation and characterization of silicate mineralsolubilizing Bacillus globisporus Q12 from the surfaces of weathered feldspar. Can J Microbiol. 2008. Vol.54. No.12. P.1064-1068.

[46] I.A. Berg. Ecological Aspects of the Distribution of Different Autotrophic $\mathrm{CO}_{2}$ Fixation Pathways. Applied And Environmental Microbiology. 2011. Vol.77. No.6. P.1925-1936.

[47] T.J. Erb. Carboxylases in Natural and Synthetic Microbial Pathways. Applied And Environmental Microbiology. 2011. Vol.77. No.24. P.8466-8477.

[48] P.V. Welander, W.W. Metcalf. Loss of the mtr operon in Methanosarcina blocks growth on methanol, but not methanogenesis, and reveals an unknown methanogenic pathway. PNAS.2005. Vol.102. No.30. P.10664-10669.

[49] F. Omil, D. Méndez, G. Vidal, R. Méndez, J. M. Lema. Biodegradation of formaldehyde under anaerobic conditions. Enzyme and Microbial Technology.1999. Vol.24. No.5-6. P.255-262.

[50] X. Yin,W. Wu, M. Maeke, T. Richter-Heitmann,A.C. Kulkarni, O.E. Oni,J. Wendt,M. Elvert, M.W. Friedrich. $\mathrm{CO}_{2}$ conversion to methane and biomass in obligate methylotrophic methanogens in marine sediments. The ISME Journal. 2019. Vol.13. No.8. P.2107-2119.

[51] K. Aoki, T. Uemori, R. Shinke, H.N. Ishira. Further Characterization of Bacterial Production of Anthranilic Acid from Aniline. Agric. BioI. Chem. 1985. Vol.49. No.4. P.1151-1158.

[52] M. Mujahid, Ch. Sasikala, Ch.V. Ramana. Aniline-Induced Tryptophan Production and Identification of Indole Derivatives from Three Purple Bacteria. Curr Microbiol. 2010. Vol.61. No.4. P.285-290.

[53] P.K. Arora. Bacterial degradation of monocyclic aromatic amines. Front. Microbiol. 2015. Vol.6. No.820. P.1-14.

[54] C.T. Walsh, S.W. Haynes, B.D. Ames. Aminobenzoates as building blocks for natural product assembly lines. Nat. Prod. Rep. 2012. Vol.29. No.1. P.37-59.

[55] T.P.T. Nguyen, C.F. Clarke. Folate status of gut microbiome affects Caenorhabditis elegans lifespan. BMC Biology.2012. Vol.10. No.66. P.1-4.

[56] A. Lallement, L. Besaury, E. Tixier, M. Sancelme, P. Amato, V. Vinatier, I. Canet, O.V. Polyakova, V.B. Artaev, A.T. Lebedev, L. Deguillaume, G. Mailhot, A.-M. Delort. Potential for phenol biodegradation in cloud waters. Biogeosciences. 2018. Vol.15. P.5733-5744.

[57] A.Comte, P.Christen, S.Davidson, M.Pophillat, J.Lorquin, R.Auria, G.Simon, L.Casalot. Biochemical, Transcriptional and Translational Evidences of the Phenol-meta-Degradation Pathway by the Hyperthermophilic Sulfolobus solfataricus 98/2. PLoSONE. 2013. Vol.8. No.12. e82397. 
METABOLISM OF PHOSPHORUS COMPOUNDSAND TAXONOMIC POSITION...

[58] A. Lack, G. Fuchs. Carboxylation of Phenylphosphate by Phenol Carboxylase, an Enzyme System of Anaerobic Phenol Metabolism. Journal of bacteriology. 1992. Vol.174. No.11. P.3629-3636.

[59] M.M. Palcic, S.-J. Shen, E. Schleicher, H. Kumagai, S. Sawada, H. Yamada, H.G. Floss. Stereochemistry and Mechanism of Reactions Catalyzed by Tyrosine Phenol-Lyase from Escherichia intermedia. Z. Naturforsch. 1987. Vol.42. No.2. P.307-318.

[60] K. Kim, P.A. Cole. Synthesis of (2S,3R)-ß-methyltyrosine catalyzed by tyrosine phenol-lyase. Bioorganic \& Medicinal Chemistry Letters. 1999. Vol.9. No.8. P.1205-1208.

[61] H. Yamada, H. Kumagai. Synthesis of L-Tyrosine-Related Amino Acids by $\beta$-Tyrosinase.Advances in Applied Microbiology. 1975. Vol.19. P.249-288.

[62] T.K. Lee, H.-y. Hsiao. Synthesis of L-tyrosine by a coupled reaction of serine hydroxymethyltransferase and $\beta$-tyrosinase. Enzyme and Microbial Technology. 1986. Vol.8. No.9. P.523-526.

[63] H. Enei, H. Matsui, H. Nakazawa, S. Okumura, H. Yamada. Synthesis of L-Tyrosine or 3, 4Dihydroxyphellyl-L-alanine from DL-Serine and Phenol or Pyrocathechol. Agr. Biol. Chem. 1973. Vol.37. No.3. P.493-499.

[64] D.R. Boyd, J.T.G. Hamilton, N.D. Sharma, J.S. Harrison, W.C. McRoberts, D.B. Harper. Isolation of a stable benzene oxide from a fungal biotransformation and evidence for an 'NIH shift' of the carbomethoxy group during hydroxylation of methyl benzoates. Chem. Commun. 2000. P.1481-1482.

[65] R. Snyder, C.C. Hedli. An Overview of Benzene Metabolism. Environmental Health Perspectives. 1996. Vol.104. No.6. P.1165-1171.

[66] M.E. Caldwell, J.M. Suflita. Detection of Phenol and Benzoate as Intermediates of Anaerobic Benzene Biodegradation under Different Terminal Electron-Accepting Conditions. Environ. Sci. Technol. 2000. Vol. 34. No.7. P.1216-1220.

[67] S.A.B. Weelink, M.H.A. van Eekert, A.J.M. Stams. Degradation of BTEX by anaerobic bacteria: physiology and application. Rev Environ Sci Biotechnol. 2010. Vol.9. No.4. P.359-385.

[68] H. Platen, B. Schink. Enzymes involved in anaerobic degradation of acetone by a denitrifying bacterium. Biodegradation. 1990. Vol.1. No.4. P.243-251.

[69] H. Iwaki, K. Abe, Y. Hasegawa. Isolation and characterization of a new 2,4-dinitrophenol - degrading bacterium Burkholderia sp. strain KU-46 and its degradation pathway. FEMS Microbiol Lett. 2007.Vol.274. No.1. P.112-117.

[70] Y.J. Rhee, S. Hillier, G.M. Gadd. Lead Transformation to Pyromorphite by Fungi. Current Biology. 2012. Vol.22. No.3. P.237-241.

[71] A. Ceci,M. Kierans,S. Hillier,A.M. Persiani,G.M. Gadd. Fungal Bioweathering of Mimetite and a General Geomycological Model for Lead Apatite Mineral Biotransformations.Appl Environ Microbiol. 2015. Vol.81. No.15. P.4955-4964.

[72] B. Antizar-Ladislao. Environmental levels, toxicity and human exposure to tributyltin (TBT)-contaminated marine environment. A review. Environment International. 2008. Vol.34. No.2. P.292-308.

[73] J. Lafrance-Vanasse, M. Lefebvre, P. Di Lello, J. Sygusch, J.G. Omichinski. Crystal Structures of the Organomercurial Lyase MerB in Its Free and Mercury-bound Forms. Insights into the mechanism of methylmercury degradation. The journal of biological chemistry. 2009. Vol.284. No.2. P.938-944.

[74] N. Mergelov, C.W. Mueller, I. Prater, I. Shorkunov, A. Dolgikh, E. Zazovskaya, V. Shishkov, V. Krupskaya, K. Abrosimov, A. Cherkinsky, S. Goryachkin. Alteration of rocks by endolithic organisms is one of the pathways for the beginning of soils on Earth. Sci. Rep. 2018. Vol.8. No.3367. P.1-15.

[75] A. Dance. Inner Workings: Endoliths hunker down and survive in extreme environments. PNAS. 2015. Vol.112. No.8. P.2296.

[76] J. Marlow, J. Peckmann, V. Orphan. Autoendoliths: a distinct type of rock-hosted microbial life. Geobiology. 2015. Vol.13. No.4. P.303-307.

[77] M. Ivarsson, H. Skogby, B. Phichaikamjornwut, S. Bengtson, S. Siljeström, P. Ounchanum, A. Boonsoong, M. Kruachanta, F. Marone, V. Belivanova, S. Holmström. Intricate tunnels in garnets from soils and river sediments in Thailand - Possible endolithic microborings. PLoS ONE. 2018. Vol.13(8). No.e0200351. P.1-20.

[78] Z. Liu, T. Fu, C. Hu, D. Shen, N. Macchioni, L. Sozzi, Y. Chen, J. Liu, X. Tian, Q. Ge, Z. Feng, H. Liu, Z. Zhang, J. Pan. Microbial community analysis and biodeterioration of waterlogged archaeological wood from the Nanhai No. 1 shipwreck during storage. Sci.Rep. 2018. Vol.8. No.7170. P.1-11.

[79] J. Biswas, K. Sharma, K.K. Harris, Y. Rajput. Biodeterioration agents: Bacterial and fungal diversity dwelling in or on the pre-historic rock-paints of Kabra-pahad, India. IJM. 2013. Vol.5. No.3. P.309-314.

[80] K. Nüsslein, D. Maris, K. Timmis, D.F. Dwyer. Expression and Transfer of Engineered Catabolic Pathways Harbored by Pseudomonas spp. Introduced into Activated Sludge Microcosms. Appl EnvironMicrobiol. 1992. Vol.58. No.10. P.3380-3386. 
[81] M. Aldas, A. Paladines, V. Valle, M. Pazmico, F. Quiroz. Effect of the Prodegradant-Additive Plastics Incorporated on the Polyethylene Recycling. International Journal of Polymer Science. 2018. Vol.2018. No.2474176. P.1-10.

[82] L. Lebreton, B. Slat, F. Ferrari, B. Sainte-Rose, J. Aitken, R. Marthouse, S. Hajbane, S. Cunsolo, A. Schwarz, A. Levivier, K. Noble, P. Debeljak, H. Maral, R. Schoeneich-Argent, R. Brambini, J. Reisser. Evidence that the Great Pacific Garbage Patch is rapidly accumulating plastic. Sci Rep. 2018. Vol.8. No.4666. P.1-15.

[83] A. Trincone, V. Lanzotti, B. Nicolaus, W. Zillig, M. De Rosa, A. Gambacorta. Comparative Lipid Composition of Aerobically and Anaerobically Grown Desulfurolobus ambivalens, an Autotrophic Thermophilic Archaeobacterium. Journal of General Microbiology. 1989. Vol.135. P.2751-2757.

[84] J.A. Baccile, J.E. Spraker, H.H. Le, E. Brandenburger, C. Gomez, J.W. Bok, J. Macheleidt, A.A. Brakhage, D. Hoffmeister, N.P. Keller, F.C. Schroeder . Plant-like biosynthesis of isoquinoline alkaloids in Aspergillus fumigatus.Nature Chemical Biology. 2016. Vol.12. No.6. P.419-424.

[85] M. Pelizzola, J.R. Ecker. The DNA methylome. FEBS Letters. 2011. Vol.585. No.13. P.1994-2000.

[86] F. Marlétaz, P.N. Firbas, I. Maeso, J.J. Tena, O. Bogdanovic, M. Perry, C.D.R. Wyatt, E. de la CalleMustienes, S. Bertrand, D. Burguera, R.D. Acemel, S.J. van Heeringen, S. Naranjo, C. Herrera-Ubeda, K. Skvortsova, S. Jimenez-Gancedo, D. Aldea, Y. Marquez, L. Buono, I. Kozmikova, J. Permanyer, A. Louis, B. Albuixech-Crespo, Y. Le Petillon, A. Leon, L. Subirana, P.J. Balwierz, P.E. Duckett, E. Farahani, J.-M. Aury, S. Mangenot, P. Wincker, R. Albalat, Ė. Benito-Gutiérrez, C. Cañestro, F. Castro, S. D’Aniello, D.E.K. Ferrier, S. Huang, V. Laudet, G.A.B. Marais, P. Pontarotti, M. Schubert, H. Seitz, I. Somorjai, T. Takahashi, O. Mirabeau, A. Xu, J.-K. Yu, P. Carninci, J.R. Martinez-Morales, H.R. Crollius, Z. Kozmik, M.T. Weirauch, J. Garcia-Fernàndez, R. Lister, B. Lenhard, P.W.H. Holland, H. Escriva, J.L. Gómez-Skarmeta, M. Irimia. Amphioxus functional genomics and the origins of vertebrate gene regulation. Nature. 2018. Vol.564. No.7734. P.64-70.

[87] B. Huber, P. Dammann, C. Krüger, P. Kirsch, B. Bialek, R.A. Diaz-Bone, R. Hensel. Production of Toxic Volatile Trimethylbismuth by the Intestinal Microbiota of Mice. Journal of Toxicology. 2011. Vol.2011. P.1-5.

[88] D.G, Knorre, C.D. Myzina. Biological chemistry. Moscow: Nauka. 479p. 2002. (russian)

[89] L. Ding, S. Fotso, F. Li, S. Qin, H. Laatsch. Hualyzin, a Symmetrical Urea Derivative Isolated from Penicillium herquei Isolate GA4. J. Nat. Prod. 2008. Vol.71. No.6. P.1068-1069.

[90] A. van der Vliet, P.A. Chr. 't Hoen, P. S.-Y. Wong, A. Basti, C.E. Cross. Formation of S-Nitrosothiols via Direct Nucleophilic Nitrosation of Thiols by Peroxynitrite with Elimination of Hydrogen Peroxide. The Journal Of Biological Chemistry.1998. Vol.273. No.46. P.30255-30262.

[91] B.J. Novak, D.R. Blake, S. Meinardi, F. S. Rowland, A. Pontello, D. M. Cooper, P.R. Galassetti. Exhaled methyl nitrate as a noninvasive marker of hyperglycemia in type 1 diabetes. PNAS .2007. Vol.104. No.40. P.15613-15618.

[92] M.L. Kopka, C. Yoon, D. Goodsell, P. Pjura, R.E. Dickerson. The molecular origin of DNA-drug specificity in netropsin and distamycin. Proc. Nati. Acad. Sci. USA. 1985.Vol.82. No.5. P.1376-1380.

[93] A. Vikram, B. Prithiviraj, A.C. Kushalappa. Use of volatile metabolite profiles to discriminate fungal diseases of cortland and empire apples. Journal of Plant Pathology.2004. Vol.86. No.3. P.215-225.

[94] D.J. Hawranik, J.L. Sorensen. The isolation of citric acid derivatives from Aspergillus niger. FEMS Microbiol Lett. 2010. Vol.306. No.2. P.122-126.

[95] I.C. Piña, J.T. Gautschi, G.-Y.-S. Wang, M.L. Sanders, F.J. Schmitz, D. France, S. Cornell-Kennon, L.C. Sambucetti, S.W. Remiszewski, L.B. Perez, K.W. Bair, P. Crews. Psammaplins from the Sponge Pseudoceratina purpurea: Inhibition of Both Histone Deacetylase and DNA Methyltransferase. J. Org. Chem. 2003. Vol.68. No.10. P.3866-3873.

[96] H. Zhang, H.-B. Liu, J.-M. Yue. Organic Carbonates from Natural Sources. Chem. Rev. 2014. Vol.114. P.883-898.

[97] S. Weinitschke, P.I. Sharma, U. Stingl, A.M. Cook, T.H.M. Smits. Gene Clusters Involved in Isethionate Degradation by Terrestrial and Marine Bacteria. Applied And Environmental Microbiology. 2010. Vol.76. No.2. P.618-621.

[98] Z. Krejčík, K. Denger, S. Weinitschke, K. Hollemeyer, V. Pačes, A.M. Cook, T.H.M. Smits. Sulfoacetate released during the assimilation of taurine-nitrogen by Neptuniibacter caesariensis: purification of sulfoacetaldehyde dehydrogenase. Arch Microbiol. 2008. Vol.190. No.2. P.159-168.

[99] M.-A. Le Pape, J. Grua-Priol, C. Prost, M Demaimay. Optimization of Dynamic Headspace Extraction of the Edible Red Algae Palmaria palmata and Identification of the Volatile Components. J. Agric. Food Chem. 2004. Vol.52. No.3. P.550-556. 
[100] C. Parkouda, B. Diawara, S. Lowor, C. Diako, F.K. Saalia, N.T. Annan, J.S. Jensen, K. Tano-Debrah, M. Jakobsen. Volatile compounds of maari, a fermented product from baobab (Adansonia digitata L.) seeds. African Journal of Biotechnology.2011. Vol.10. No.20.P.4197-4206.

[101] J.E. Balkus, C. Mitchell, K. Agnew, C. Liu, T. Fiedler, S.E. Cohn, A. Luque, R. Coombs, D.N. Fredricks, J. Hitt. Detection of hydrogen peroxide-producing Lactobacillus species in the vagina: a comparison of culture and quantitative PCR among HIV-1 seropositive women. BMC Infectious Diseases. 2012. Vol.12. No.188. P.1-5.

[102] C.-K. Wen, editor. Ethylene in Plants. Springer. 2015. 286p.

[103] C. Calfapietra, S. Fares, F. Manes, A. Morani, G. Sgrigna, F. Loreto. Role of Biogenic Volatile Organic Compounds (BVOC) emitted by urban trees on ozone concentration in cities: A review. Environmental Pollution. 2013. Vol.183. P.71-80.

[104] J. Zhang, S. Sturla, C. Lacroix, C. Schwab. Gut Microbial Glycerol Metabolism as an Endogenous Acrolein Source. American Society of Microbiology. 2018. Vol.9.No.1 e01947-17. P.1-6.

[105] I. Ujváry. Unnatural and seminatural products as alleged natural pesticides from plants. Conference: XIVth International Plant Protection Congress Plant Protection Towards the Third Millennium - Where Chemistry Meets Ecology. Jerusalem, 1999.

[106] J.-F. Lagorce, J.-C.Thomes, G. Catanzano, J. Buxeraud, M. Raby, C. Raby. Formation of molecular iodine during oxidation of iodide by the peroxidase $/ \mathrm{H}_{2} \mathrm{O}_{2}$ system: Implications for antithyroid therapy. Biochem Pharmacol. 1991. Vol.42.No.1. P.S89-S92.

[107] G. Crini. Review: A History of Cyclodextrins. Chem. Rev. 2014. Vol.114. No.21. P.10940-10975.

[108] H.-K. Kang, H. Cha, T.-J. Yang, J.-T. Park, S. Lee, Y.-W. Kim, J.-H. Auh, Y. Okada, J.-W.Kim, J. Cha, C. H. Kim, K.-H. Park. Enzymatic synthesis of dimaltosyl- $\beta$-cyclodextrin via a transglycosylation reaction using TreX, a Sulfolobus solfataricus P2 debranching enzyme. Biochemical and Biophysical Research Communications. 2008. Vol.366. No.1. P.98-103.

[109] C.J. Le Coz, G. Ducombs, E. Paulsen. Plants and Plant Products. Contact Dermatitis. Editors D. Johansen, P.J. Frosch, J.-P. Lepoittevin. Chapter 46. Springer. 2011. P.873-925.

[110] M. Brückmann, R. Blasco, K.N. Timmis, D.H. Pieper. Detoxification of Protoanemonin by Dienelactone Hydrolase. Journal of Bacteriology. 1998. Vol.180. No.2. P.400-402.

[111] Y. Kuwahara, H. Ômura, T. Tanabe. 2-Nitroethenylbenzenes as natural products in millipede defense secretions. Naturwissenschaften. 2002. Vol.89. No.7. P.308-310.

[112] M. Namikoshi, T. Fujiwara, T. Nishikawa, K. Ukai. Natural Abundance 14C Content of Dibutyl Phthalate (DBP) from Three Marine Algae. Mar. Drugs. 2006. Vol.4. No.4. P.290-297.

[113] P.J. Stabler, P.J. Holt, N.C. Bruce. Transformation of 2,29-Bimorphine to the Novel Compounds 10$\alpha$-S-Monohydroxy-2,2'-Bimorphine and 10,10'- $\alpha, \alpha^{\prime}-S, S$ '- Dihydroxy-2,2'-Bimorphine by Cylindrocarpon didymium. Appl. Environ. Microbiol. 2001. Vol.67. No.8.P.3716-3719.

[114] M.T. Long, A.M. Hailes, G.W. Kirby, N.C. Bruce. Transformations of Morphine Alkaloids by Pseudomonas putida M10. Appl. Environ. Microbiol. 1995. Vol.61. No.10. P.3645-3649.

[115] S. Niknam, M.A. Faramarzi, K. Abdi, M. T. Yazdi, M. Amini, H. Rastegar. Bioconversion of codeine to semi-synthetic opiate derivatives by the cyanobacterium Nostoc muscorum. World J Microbiol Biotechnol.2010. Vol.26. No.1. P.119-123.

[116] D.L. Lister, G. Kanungo, D.A. Rathbone, N.C. Bruce. Transformations of codeine to important semisynthetic opiate derivatives by Pseudomonas putida m10. FEMS Microbiology Letters.1999. Vol.181. No.1. P.137-144.

[117] S. Morimoto, K. Suemori, F. Taura, Y. Shoyama. New Dimeric Morphine from Opium Poppy (Papaver somuniferum) and Its Physiological Function.J. Nat. Prod.2003. Vol.66. No.7. P.987-989.

[118] M.T.H. Khan, A. Ather. Microbial Transformation of Nitrogenous Compounds. Top Heterocycl Chem. 2007. Vol.10. P.99-122.

[119] H. Rashidi, M.T. Akhtar, F. van der Kooy, R. Verpoorte, W.A. Duetz. Hydroxylation and Further Oxidation of $\Delta^{9}$-Tetrahydrocannabinol by Alkane-Degrading Bacteria. Applied and environmental microbiology.2009. Vol.75. No.22. P.7135-7141.

[120] A.J. Wargacki, E. Leonard, M.N. Win, D.D. Regitsky, C.N.S. Santos, P.B. Kim, S.R. Cooper, R.M. Raisner, A. Herman, A.B. Sivitz, A. Lakshmanaswamy, Y. Kashiyama, D. Baker, Y. Yoshikuni1. An Engineered Microbial Platform for Direct Biofuel Production from Brown Macroalgae. Science.2012. Vol.335. No.6066. P.308-313.

[121] Z. Csanádi, R. Kurdi, K. Bélafi-Bakó. Ethyl-acetate synthesis in gas phase by immobilised lipase. Hungarian Journal Of Industry And Chemistry Veszprém. 2012. Vol.40. No.1. P.39-44. 
[122] S. Jamwal, R. Dharela, R. Gupta, J.-H. Ahn, G.S. Chauhan. Synthesis of crosslinked lipase aggregates and their use in the synthesis of aspirin. Chemical Engineering Research \& Design. 2015. Vol.97. P.159-164.

[123] C.K. Naso-Kaspar, J.F. Wyman, C.R. Clark, L.R. Glass, E.S. Lavins, C.E. Cushman, K.L. Pekarski, T.P. Gilson. In Vitro Formation of Acetylmorphine from Morphine and Aspirin in Postmortem Gastric Contents and Deionized Water. Journal of Analytical Toxicology. 2013. Vol.37. No.8. P.500-506.

[124] M. Božič, V. Vivoda, S. Kavčič, M. Leitgeb, V. Kokol. New findings about the lipase acetylation of nanofibrillated cellulose using acetic anhydride as acyl donor. Carbohydrate Polymers. 2015. Vol.125. P.340-351.

[125] P. Skoczinski, M.K. Espinoza Cangahuala, D. Maniar, K. Loos. Lipase-Catalyzed Transamidation of Urethane-Bond-Containing Ester. ACS Omega. 2020. Vol.5. No.3. P.1488-1495.

[126] S. Matsumura, Y. Soeda, K. Toshima. Perspectives for synthesis and production of polyurethanes and related polymers by enzymes directed toward green and sustainable chemistry. Appl Microbiol Biotechnol. 2006. Vol.70. No.1. P.12-20.

[127] H.N. Cheng, Q.-M. Gu. Enzyme-Catalyzed Modifications of Polysaccharides and Poly(ethylene glycol). Polymers. 2012. Vol.4. No.4. P.1311-1330.

[128] S. Kobayashi. Lipase-catalyzed polyester synthesis - A green polymer chemistry. Proc. Jpn. Acad., Ser. B. 2010. Vol.86. No.4. P.338-365.

[129] D. Maniar,Y. Jiang, A. J.J. Woortman, J. van Dijken, K. Loos. Furan-Based Copolyesters from Renewable Resources: Enzymatic Synthesis and Properties. ChemSusChem. 2019. Vol.12. No.5. P.990-999.

[130] J. Feng, R. Zhuo, F. He, X. Wang. Synthesis of functional polycarbonates by lipase-catalyzed ringopening polymerization. Macromolecular Symposia. 2003. Vol.195. No.1. P.237-240.

[131] T.F. Al-Azemi, K.S. Bisht. Novel Functional Polycarbonate by Lipase-Catalyzed Ring-Opening Polymerization of 5-Methyl-5-benzyloxycarbonyl-1,3-dioxan-2-one. Macromolecules. 1999. Vol.32. No.20. P.6536-6540.

[132] Y. Osanai, K. Toshima, S. Matsumura. Enzymatic transformation of aliphatic polyesters into cyclic oligomers using enzyme packed column under continuous flow of supercritical carbon dioxide with toluene. Science and Technology of Advanced Materials. 2006. Vol.7. No.2. P.202-208.

[133] P.I. Nikel, V. de Lorenzo. Pseudomonas putida as a functional chassis for industrial biocatalysis: From native biochemistry to trans-metabolism. Metabolic Engineering. 2018. Vol.50. P.142-155.

[134] J. Sun, Q. Wang, Y. Jiang, Z. Wen, L. Yang, J. Wu, S. Yang. Genome editing and transcriptional repression in Pseudomonas putida KT2440 via the type II CRISPR system. Microb Cell Fact. 2018. Vol.17. No.41. P.1-17.

[135] H. Zanker, G. Lurz, U. Langridge, P. Langridge, D. Kreusch, J. Schröder. Octopine and Nopaline Oxidases from Ti Plasmids of Agrobacterium tumefaciens: Molecular Analysis, Relationship, and Functional Characterization. Journal of Bacteriology. 1994. Vol.176. No.15. P.4511-4517.

[136] A.Z. Mindubaev, J.A. Akosah, F.K. Alimova, D.M.Afordoanyi, R.M. Kagirov, S.T. Minzanova, L.G. Mironova, D.G. Yakhvarov. On the White Phosphorus Degradation by Wastewater Mud. Scientific Notes of Kazan University. Natural Science Serie. 2011. Vol.153. No.2. P.110-119. (russian)

[137] A.Z. Mindubaev, F.K. Alimova, S.C. Ahossiyenagbe, C. Bolormaa, A.D. Voloshina, N.V. Kulik, S.T. Minzanova, L.G. Mironova, D.G. Yakhvarov. The possibility for anaerobicdetoxicationof white phosphorus. Butlerov Communications. 2013. Vol.33. No.1. P.22-34. ROI: jbc-02/13-33-1-22

[138] A.Z. Mindubaev, A.D. Voloshina, D.G. Yakhvarov. Biological degradation of white phosphorus: feasibility and prospects.Butlerov Communications. 2013. Vol.33. No.2. P.1-17.ROI: jbc-02/13-33-2-1

[139] A.Z. Mindubaev, F.K. Alimova, S.C. Ahossiyenagbe, S.T. Minzanova, L.G. Mironova, D.G. Yakhvarov. New confirmation for white phosphorus biodegradation. Butlerov Communications. 2013. Vol.36. No.10. P.1-12. ROI: jbc-02/13-36-10-1

[140] A.Z. Mindubaev, F.K. Alimova, S.C. Ahossiyenagbe, C. Bolormaa, A.D. Voloshina, N.V. Kulik, S.T. Minzanova, L.G.Mironova,D.G. Yakhvarov. Microbial metabolism of the white phosphorus. Butlerov Communications. 2013. Vol.36. No.12. P.34-52. ROI: jbc-02/13-36-12-34

[141] A.Z. Mindubaev, F.K. Alimova, S.C. Ahossiyenagbe, A.D. Voloshina, E.V. Gorbachuk, N.V. Kulik, S.T. Minzanova, L.G.Mironova,D.G. Yakhvarov. Metabolites and tolerant microflora in substrates with white phosphorus $0.1 \%$. Butlerov Communications. 2014. Vol.37. No.3. P.67-78. ROI: jbc02/14-37-3-67

[142] C. Bolormaa, K.A. Saparmyradov,F.K. Alimova, A.Z. Mindubaev. Comparison of phytotoxicity indices, fungicidal and bactericidal activity of Streptomices from different habitats. Butlerov Communications. 2014. Vol.38. No.6. P.147-152. ROI: jbc-02/14-38-6-147 
METABOLISM OF PHOSPHORUS COMPOUNDSAND TAXONOMIC POSITION...

[143] A.Z. Mindubaev, A.D. Voloshina, E.V. Gorbachuk, N.V. Kulik, S.C. Ahossiyenagbe, F.K. Alimova, S.T. Minzanova, L.G.Mironova,A.V. Pankova, C. Bolormaa, K.A. Saparmyradov, D.G. Yakhvarov. White phosphorusas a new objectof biological destruction. Butlerov Communications. 2014. Vol.40. No.12. P.1-26. ROI: jbc-02/14-40-12-1

[144] A.Z. Mindubaev, A.D. Voloshina, E.V. Gorbachuk, N.V. Kulik, F.K. Alimova, S.T. Minzanova, L.G.Mironova, K.A. Saparmyradov,K.R. Khayarov, D.G. Yakhvarov. The inclusion white phosphorusin the naturalcycle of matter. Cultivation of resistant microorganisms. Butlerov Communications. 2015. Vol.41. No.3. P.54-81. ROI: jbc-02/15-41-3-54

[145] A.Z. Mindubaev, A.D. Voloshina, E.V. Gorbachuk, S. Z. Validov,N.V. Kulik, F.K. Alimova, S.T. Minzanova, L.G.Mironova, D.E. Belostotskiy, K.A. Saparmyradov, R.I. Tukhbatova,D.G. Yakhvarov. Adaptation of microorganisms to white phosphorus as a resultof directed selection. Genetic identification of sustainable Aspergillus and metabolic profiling of Streptomyces A8.Butlerov Communications. 2015. Vol.44. No.12. P.1-28. DOI: 10.37952/ROI-jbc-01/15-44-12-1

[146] A.Z. Mindubaev. Biodegradation of white phosphorus: how a poison has become a fertilizer. Biomolekula. 2016. http://biomolecula.ru/content/1932

[147] A.Z. Mindubaev, A.D. Voloshina, S. Z. Validov, N.V. Kulik, S.T. Minzanova, L.G.Mironova, D.G. Yakhvarov, A.Y. Akkizov. Aspergillus niger AM1 culture growth in medium with two phosphorus sources. The validity of the definition"biodegradation" with respect to white phosphorus. Butlerov Communications. 2016. Vol.46. No.5. P.1-20. DOI: 10.37952/ROI-jbc-01/16-46-5-1

[148] A.Z. Mindubaev, F.K. Alimova, A.D. Voloshina, E.V. Gorbachuk, N.V. Kulik, S.T. Minzanova, R.I. Tukhbatova, D.G. Yakhvarov. Method for detoxification of white phosphorus using microorganism strain Trichoderma asperellum VKPM F-1087. Patent RF No 2603259 from 1.11.2016. Bul. 33. Reciprocity date 28. 07. 2015. Registration number 2015131380 (048333). The decision to grant a patent on 29. 08. 2016. (russian)

[149] A.Z. Mindubaev, E.V. Babynin, A.D. Voloshina, S.Z. Validov, N.V. Kulik, S.T. Minzanova, L.G.Mironova, A.Y. Akkizov,D.G. Yakhvarov. Evaluation of white phosphorus genotoxicity. Growth of bacterial culture in a medium with potassium phosphite as a sole source of phosphorus. Butlerov Communications. 2016. Vol.47. No.7. P.1-20. DOI: 10.37952/ROI-jbc-01/16-47-7-1

[150] A.Z. Mindubaev, E.V. Babynin, A.D. Voloshina, I.F. Sakhapov, N.V. Kulik, S.Z. Validov, S.T. Minzanova, L.G.Mironova, A. Y. Akkizov, D.G. Yakhvarov. Genotoxicity ofwhitephosphorus. Butlerov Communications. 2017. Vol.49. No.1. P.1-20. DOI: 10.37952/ROI-jbc-01/17-49-1-1

[151] A.Z. Mindubaev, A.D. Voloshina, S. Z. Validov, N.V. Kulik, S.T. Minzanova, L.G.Mironova, D.G. Yakhvarov, A.Y. Akkizov. Aspergillus niger AM1 culture growth in medium with two phosphorus sources. The validity of the definition "biodegradation" with respect to white phosphorus. Butlerov Communications. 2016. Vol.46. No.5. P.1-20. DOI: 10.37952/ROI-jbc-01/16-46-5-1

[152] A.Z. Mindubaev, A.D. Voloshina, Sh.Z. Validov, D.G. Yakhvarov. Biodegradation of white phosphorus. Priroda. 2017. No.5. P.29-43. (russian)

[153] A.Z. Mindubaev, A.D. Voloshina, E.V. Gorbachuk, S.Z. Validov, N.V. Kulik, F.K. Alimova, S.T. Minzanova, L.G. Mironova, D.E. Belostotsky, K.A. Saparmyradov, R.I. Tukhbatova, D.G.Yakhvarov. Adaptation of microorganisms to white phosphorus as a result of directed selection. Genetic identification of sustainable Aspergillus and metabolic profiling of Streptomyces A8. Butlerov Communications. 2015. Vol.44. No.12. P.1-28. DOI: 10.37952/ROI-jbc-01/15-44-12-1

[154] A.Z. Mindubaev, E.V. Babynin, S.Z. Validov, A.D. Voloshina, N.V. Kulik, S.T. Minzanova, L.G. Mironova, A.Y. Akkizov, D.G. Yakhvarov. Evaluation of white phosphorus genotoxicity. Growth of bacterial culture in a medium with potassium phosphite as a sole source of phosphorus. Butlerov Communications. 2016. Vol.47. No.7. P.1-20. DOI: 10.37952/ROI-jbc-01/16-47-7-1

[155] A.Z. Mindubaev, E.V. Babynin, A.D. Voloshina, I.F. Sakhapov, N.V. Kulik, S.Z. Validov, S.T. Minzanova, L.G. Mironova, A.Y. Akkizov, D.G. Yakhvarov. Genotoxicity of white phosphorus. Butlerov Communications. 2017. Vol.49. No.1. P.1-20. DOI: 10.37952/ROI-jbc-01/17-49-1-1

[156] A.Z. Mindubaev, A.D. Voloshina, D.G. Yakhvarov. Biological degradation of white phosphorus: feasibility and prospects. Butlerov Communications. 2013. Vol.33. No.2. P.1-17. ROI: jbc-02/13-33-2-1

[157] A.Z. Mindubaev, A.D. Voloshina, Kh.R. Khayarov, I.F. Sakhapov, E.K. Badeeva, A.S. Strobykina, Sh.Z. Validov, V.M. Babaev, S.T. Minzanova, L.G. Mironova, A.Y. Abaye, D.G. Yakhvarov. Dynamics of white phosphorus transformation by a culture of black aspergill. Butlerov Communications. 2017. Vol.51. No.8. P.1-26. DOI: 10.37952/ROI-jbc-01/17-51-8-1

[158] A.Z. Mindubaev, A.D. Voloshina, E.V. Babynin, E.K. Badeeva, Kh.R. Khayarov, S.T. Minzanova, D.G. Yakhvarov. Microbiological degradation of white phosphorus. Ecology and Industry of Russia. 2018. Vol.22. Iss.1. P.33-37. (russian) 
[159] A.Z. Mindubaev, A.D. Voloshina, E.V. Babynin, Sh.Z. Validov, K.A. Saparmyradov, Kh.R. Khayarov, E.K. Badeeva, S.T. Minzanova, L.G. Mironova, Y.A. Akosah, D.G. Yakhvarov. Neutralization of white phosphorus by means of microbiological decomposition. Butlerov Communications. 2017. Vol.52. No.12. P.87-118. DOI: 10.37952/ROI-jbc-01/17-52-12-87

[160] A.Z. Mindubaev, A.Y. Abaye, D.G. Yakhvarov. Phosphine oxide as a prospective intermediate of biological processes. Butlerov Communications. 2018. Vol.53. No.3. P.1-34. DOI: 10.37952/ROI-jbc01/18-53-3-1

[161] A.Z. Mindubaev, A.D. Voloshina, E.V. Babynin, Sh.Z. Validov, K.A. Saparmyradov, Kh.R. Khayarov, E.K. Badeeva, S.T. Minzanova, L.G.Mironova, Y.A. Akosah, D.G. Yakhvarov. Neutralization of white phosphorus by means of microbiological decomposition. Butlerov Communications. 2017. Vol.52. No.12. P.87-118. DOI: 10.37952/ROI-jbc-01/17-52-12-87

[162] A.Z. Mindubaev, E.V. Babynin, D.B. Piskunov, A.N. Makhiyanov, E.K. Badeeva, S.T. Minzanova, L.G. Mironova, A.Y. Abayie, A.D. Voloshina. Cytogenetic effect of white phosphorus. Butlerov Communications. 2018. Vol.55. No.9. P.1-21. DOI: 10.37952/ROI-jbc-01/18-55-9-1

[163] A.Z. Mindubaev, A.D. Voloshina, E.V. Babynin, S.T. Minzanova, L.G. Mironova, E.K. Badeeva. Investigating the biodegradation of white phosphorus. Proceedings of the II International Scientific Conference "Problems of Environmental Education in the XXI Century". Vladimir. 2018. P.172-176. (russian)

[164] A.Z. Mindubaev, E.V. Babynin, E.K. Badeeva, G.G. Kabirova, A.A. Sinitsina, R.K. Shaykhutdinov, A.A. Sharipov, S.T. Minzanova, L.G. Mironova, Y.A. Akosah. The resistance of Aspergillus niger strains and bacteria to white phosphorus. The impact of divalent copper on biodegradation. Butlerov Communications. 2018. Vol.56. No.11. P.1-24. DOI: 10.37952/ROI-jbc-01/18-56-11-1

[165] A.Z. Mindubaev. From a poison to a fertilizer. Science and Life. 2019. No.3. P.46-47. (russian)

[166] A.Z. Mindubaev, E.V. Babynin, A.D. Voloshina, Kh.R. Khayarov, E.K. Badeeva, S.T. Minzanova, L.G. Mironova, Ya.A. Akosah. A rational for the method of biological purification of environments contaminated with white phosphorus. Belgorod State University Scientific Bulletin. Natural sciences series. 2019. Vol.43. No.1. P.87-97. (russian) DOI 10.18413/2075-4671-2019-43-1-87-97

[167] A. Z. Mindubaev, E.V. Babynin, D.B. Piskunov, A.N. Makhiyanov, E.K. Badeeva, S.T. Minzanova, L.G. Mironova, A.D. Voloshina. Genotoxicity and cytogenetic effect of white phosphorus. Proceedings of Universities. Applied Chemistry and Biotechnology. 2019. Vol.9. No.1. P.81-94. (russian). DOI: http://dx.doi.org/10.21285/2227-2925-2019-9-1-81-94

[168] A.Z. Mindubaev, E.V. Babynin, E.K. Badeeva, S.T. Minzanova, L.G. Mironova,Y.A. Akosah. The influence of the culture media composition on the white phosphorus biodegradation by Aspergillus niger. Butlerov Communications. 2019. Vol.58. No.5. P.1-23. DOI: 10.37952/ROI-jbc-01/19-58-5-1

[169] A.Z. Mindubaev, E.V. Babynin, A.D.Voloshina, K.A. Saparmyradov, Y.A. Akosah, E.K. Badeeva, S.T. Minzanova, L.G. Mironova. The possibility of neutralizing white phosphorus using microbial cultures. News of NAS RK. Series of geology and technical sciences. 2019. Vol.5. No.437. P.122-128. https://doi.org/10.32014/2019.2518-1491.63

[170] A.Z. Mindubaev, E.V. Babynin, A.D.Voloshina, Kh.R. Khayarov, E.K. Badeeva, S.T. Minzanova, L.G. Mironova. Study of white phosphorus biological detoxication. Proceedings of national polytechnic university of Armenia. Chemical and environmental technologies. 2018. No.2. P.9-20. (russian)

[171] A.Z. Mindubaev, A.D. Voloshina, Y.A. Akosah. Neutralization of white phosphorus by fungal culture. Сборник тезисов пятой международной научно-практической конференции «Оиенка воздействия отходов на окружающую среду административного района, очистка и обработка». Ереван (Армения). 2019.С.12-13.

[172] A.Z. Mindubaev, A.D. Voloshina, N.V. Kulik, T.A. Barsukova, Y.A. Akosah, S.T. Minzanova, L.G. Mironova The possible clearance of white phosphorus pollution using sewage sludge microbes.

Biomics. 2019. Vol.11. No.4. P. Vol.11. No.4. P.402-408. (russian). DOI: 10.31301/22216197.bmcs.2019-32

[173] A.Z. Mindubaev, A.D.Voloshina, S.T. Minzanova, E.K. Badeeva. Detoxication of wastewaters containing white phosphorus using microbiota. Science in the regional space of modern Russia and foreign countries. Syktyvkar. 2019. P.206-210. DOI: 10.19110/7934-002

[174] A.Z. Mindubaev, E.V. Babynin, A.D. Voloshina, E.K. Badeeva, S.T. Minzanova, L.G. Mironova. Disposal of first class hazards eco toxicants white and red phosphorus possibility. XXI century. Technosphere Safety. 2019. Vol.4. No.4. P.410-421. (russian) 
METABOLISM OF PHOSPHORUS COMPOUNDSAND TAXONOMIC POSITION...

[175] A.Z. Mindubaev, A.D. Voloshina, E.V. Babynin, S.T. Minzanova, L.G. Mironova, K.A. Saparmyradov, E.K. Badeeva. Study of detoxification of white (yellow) phosphorus by microbial strains. (russian)

[176] A.Z. Mindubaev, A.D. Voloshina, N.V. Kulik, D.V. Ryzhikov, T.A. Barsukova, Y.A. Akosah, S.T. Minzanova, L.G. Mironova. Biodegradation of White Phosphorus - a Dangerous Industrial Contaminant. Biogeosystem Technique. 2019. Vol.6. No.2. P.91-101. (russian). DOI: 10.13187/bgt.2019.2.91

[177] A.Z. Mindubaev, E.V. Babynin, E.K. Badeeva, S.T. Minzanova, L.G. Mironova. The Effect of Culture Media Composition and Microorganism Species Affiliation on the Biological Destruction of White Phosphorus. Biogeosystem Technique. 2019. Vol.6. No.2. P.102-113. (russian). DOI: 10.13187/bgt.2019.2.102

[178] A.Z. Mindubaev,E.V. Babynin, E.K. Badeeva, S.T. Minzanova, L.G.Mironova,I.S. Nizamov, N.R. Khasiyatullina, L.M. Pirut, E.E. Barskaya,Y.A. Akosah. Biodegradation of a phosphorus compounds by the culture of black aspergill. Butlerov Communications. 2019. Vol.60. No.12. P.1-24. DOI: 10.37952/ROI-jbc-01/19-60-12-1

[179] A.Z. Mindubaev, S.V. Kuznetsova, V.G. Evtyugin, A.G. Daminova, T.V. Grigoryeva, Y.D. Romanova, V.A. Romanova, V.M. Babaev, D.N. Buzyurova, E.V. Babynin, E.K. Badeeva, S.T. Minzanova, L.G. Mironova. Effect of White Phosphorus on the Survival, Cellular Morphology, and Proteome of Aspergillus niger. Prikladnaya Biokhimiya i Mikrobiologiya. 2020. Vol.56. No.2. P.156164. DOI 10.31857/S0555109920020117

[180] A.Z. Mindubaev, E.V. Babynin, A.D. Voloshina, S.T. Minzanova, L.G. Mironova, K.A. Saparmyradov, E.K. Badeeva, Y.A. Akosah, Kh.R. Khayarov. Investigating the biological degradation of white phosphorus. Bulletin of the Kamchatka Polytechnic College. 2019. No.21. P.10-29. (russian)

[181] A.Z. Mindubaev, E.V. Babynin, E.K. Badeeva, S.T. Minzanova. Biodegradation of phosphorus compounds by Aspergillus niger AM1.Materials of the All-Russian scientific and practical conference with international participation "Utilization of production and consumption waste: innovative approaches and technologies." Kirov. 2019. Bk.1. P.156-159. (russian)

[182] A.Z. Mindubaev, E.V. Babynin, A.D. Voloshina, E.K. Badeeva, S.T. Minzanova, L.G. Mironova, Y.A. Akosah. Biological degradation of white and red phosphorus. Academical bulletin ELPIT. 2019. Vol.4. No.4(10). P.28-44.(russian)

[183] A.Z. Mindubaev, S.V. Kuznetsova, V.G. Evtyugin, A.G. Daminova, T.V. Grigoryeva, Y.D. Romanova, V.A. Romanova, V.M. Babaev, D.N. Buzyurova, E.V. Babynin, E.K. Badeeva, S.T. Minzanova, L.G. Mironova. Effect of White Phosphorus on the Survival, Cellular Morphology, and Proteome of Aspergillus niger.Applied Biochemistry and Microbiology. 2020. Vol.56. No.2. P.194201. DOI: $10.1134 /$ S0003683820020118

[184] A.Z. Mindubaev, E.V. Babynin, E.K. Badeeva. Growth of black aspergill on a row of phosphorus compounds. The North Caucasus Ecological Herald. 2020. Vol.16. No.1. P.64-72. (russian)

[185] A.Z. Mindubaev, E.V. Babynin, S.T. Minzanova, L.G. Mironova, E.K. Badeeva. Influence of the composition of culture media on the biodegradation of white phosphorus. Biodegradation of phosphorus compounds. Collection of scientific papers based on the materials of the International Scientific Ecological Conference "Agrarian Landscapes, Their Sustainability and Development Features", Krasnodar. 2020. P.57-60. (russian)

[186] A.Z. Mindubaev, E.V. Babynin, E.K. Badeeva, S.T. Minzanova. Genotoxicity of white phosphorus for eukaryotic organisms. Collection of materials of the VIII All-Russian conference "Topical issues of chemical technology and environmental protection". Cheboksary. 2020. P.99-100. (russian)

[187] A.Z. Mindubaev, E.V. Babynin, E.K. Badeeva. The effect of divalent copper on the biodegradation of white phosphorus. Collection of materials of the IV All-Russian scientific and practical conference with international participation "Actual problems of chemistry, biotechnology and services." Irkutsk. 2020. P.110-113.(russian)

[188] A.Z. Mindubaev, E.V. Babynin, A.D. Voloshina, K.A. Saparmyradov, E.K. Badeeva, S.T. Minzanova, L.G. Mironova, Kh.R. Khayarov. Investigating the biological degradation of elemental phosphorus. Samarskaya Luka: problems of regional and global ecology. 2020. Vol.29. No.1. P.8896. (russian)

[189] A.Z. Mindubaev, E.V. Babynin, E.K. Badeeva, I.S. Nizamov, S.T. Minzanova, L.G. Mironova. Biodegradation of elemental phosphorus and phosphorus compounds by Aspergillus niger cultures. Materials of the All-Russian Scientific and Practical Conference "Theoretical and Applied Aspects of Natural Science Education". Cheboksary. 2020. P.169-177. (russian) 
[190] Y.A. Akosah, A.Z. Mindubaev, E.V. Babynin, S.T. Minzanova, E.K. Badeeva. Biological transformation of elemental phosphorus into biogenic phosphate. Materials of the Youth School, II All-Russian Conference of Young Scientists of the Agroindustrial Complex "Topical Issues of Development of Agricultural Sectors: Theory and Practice", Rassvet. 2020. P. 9-13. (russian)

[191] A.Z. Mindubaev, E.V. Babynin, E.K. Badeeva. Metabolism of phosphorus compounds by the fungus Aspergillus niger AM1. The North Caucasus Ecological Herald. 2020. Vol.16. No.2. P.45-52. (russian)

[192] Y.A. Akosah, A.Z. Mindubaev. Biodegradation of aphosphorus compounds bythe Aspergillusniger culture. Collection of materials of the XXI Professor L.P. Kuleva and N.M. Kizhner's International Scientific and Practical Conference for students and young scientists "Chemistry and chemical technology in the XXI century", Tomsk. 2020. P.507. (russian)

[193] A.Z. Mindubaev, E.V. Babynin, E.K. Badeeva, S.T. Minzanova. Biological neutralization of white phosphorus - a first class hazardous substance. Collection of materials of the VI International intramural scientific-practical conference, "Ecology of the XXI century: synthesis of education and science." Chelyabinsk. 2020. P.210-214. (russian)

[194] J.W. McGrath, J.P. Chin, J.P. Quinn. Organophosphonates revealed: new insights into the microbial metabolism of ancient molecules. Nature Reviews Microbiology. 2013. Vol.11. No.6. P.412-419.

[195] S.V. Kononova, M.A. Nesmeyanova. Phosphonates and Their Degradation by Microorganisms. Biochemistry (Moscow). 2002. Vol. 67. No.2. P.184-195. Translated from. Biokhimiya. 2002. Vol.67. No.2. P.220-233.

[196] A.Z. Mindubaev, D.G. Yakhvarov. Phosphorus: properties and application. Butlerov Communications. 2014. Vol.39. No.7. P.1-24. ROI: jbc-02/14-39-7-1

[197] G. Audi, O. Bersillon, J. Blachot, A.H. Wapstra. The NUBASE evaluation of nuclear and decay properties. Nuclear Physics A. 2003. Vol.729. No.1. P.3-128.

[198] O.B. Tarasov, D.S. Ahn, D. Bazin, N. Fukuda, A. Gade, M. Hausmann, N. Inabe, S. Ishikawa, N. Iwasa, K. Kawata, T. Komatsubara, T. Kubo, K. Kusaka, D.J. Morrissey, M. Ohtake, H. Otsu, M. Portillo, T. Sakakibara, H. Sakurai, H. Sato, B.M. Sherrill, Y. Shimizu, A. Stolz, T. Sumikama, H. Suzuki, H. Takeda, M. Thoennessen, H. Ueno, Y. Yanagisawa, K. Yoshida. Discovery of ${ }^{60} \mathrm{Ca}$ and Implications For the Stability of ${ }^{70}$ Ca. Phys. Rev. Lett. 2018. Vol.121. No.022501.

[199] J.B. Lambert, E.P. Mazzola, C.D. Ridge. Nuclear magnetic resonance spectroscopy: an introduction to principles, applications, and experimental methods. Wiley. 2019. 481p.

[200] S.M. Resnick, D.T. Gibson. Biotransformation of anisole and phenetole by aerobic hydrocarbonoxidizing bacteria. Biodegradation. 1993. Vol.4. P.195-203.

[201] K. Watanabe, N. Shimizu. Identification of a sex pheromone of the chrysanthemum lace bug Corythucha marmorata (Hemiptera: Tingidae). SciRep. 2017. Vol.7. No.7302. P.1-8.

[202] H.-L. Tsang, J.-L. Huang, Y.-H. Lin, K.-F. Huang, P.-L. Lu, G.-H. Lin, A.A. Khine, A. Hu, H.-P. Chen. Borneol Dehydrogenase from Pseudomonas sp. Strain TCU-HL1 Catalyzes the Oxidation of (+)-Borneol and Its Isomers to Camphor. Appl Environt Microbiol. 2016. Vol.82. No.21. P.63786385 .

[203] M.A. Bedewitz, E. Gyngora-Castillo, J.B. Uebler, E. Gonzales-Vigil, K.E. Wiegert-Rininger, K.L. Childs, J.P. Hamilton, B. Vaillancourt, Y.-S. Yeo, J. Chappell, D. DellaPenna, A.D. Jones, C.R. Buell, C.S. Barrya. A Root-Expressed L-Phenylalanine:4-Hydroxyphenylpyruvate Aminotransferase Is Required for Tropane Alkaloid Biosynthesis in Atropa belladonna.The Plant Cell. 2014. Vol.26. No.9. P.3745-3762.

[204] F. Sanger, S. Nicklen, A.R. Coulson. DNA sequencing with chain-terminating inhibitors. Proceedings of the National Academy of Sciences, USA. 1977. Vol.74. No.12. P.5463-5467.

[205] P.H.A. Sneath, R.R. Sokal. Numerical Taxonomy: the principals and practice of numerical classification. Freeman, San Francisco. 1973. 573p.

[206] J. Felsenstein. Confidence limits on phylogenies: An approach using the bootstrap. Evolution.1985. Vol.39. No.4. P.783-791.

[207] K. Tamura, M. Nei, S. Kumar. Prospects for inferring very large phylogenies by using the neighborjoining method. PNAS. 2004. Vol.101. No.30. P.11030-11035.

[208] T.A. Denisenko, A.B. Vishnikin, L.P. Tsiganok. Reaction features of 18-molibdodiphosphate and Folin-Ciocalteu reagent with phenolic compounds. Analitika i kontrol. 2015. Vol.19. No.3. P.242-25. (russian)

[209] Y. Segall. Biomimetic Chemistry as a Useful Tool for Studying Reactive Metabolites of Pesticides. $J$. Agric. Food Chem. 2011. Vol.59. No.7. P.2845-2856. 